\title{
Pre-treatment FLT-PET parameters as a prognostic tool for patients with locally advanced recurrent nasopharyngeal carcinoma salvaged by carbon-ion radiotherapy: a pilot study
}

\author{
Jiyi Hu ${ }^{1,2}$, Zhongyi Yang ${ }^{3,4,5,6}$, Jing Gao ${ }^{1,2}$, Weixu Hu ${ }^{1,2}$, Jing Yang ${ }^{1,2}$, Xianxin Qiu ${ }^{1,2}$, Guang $\mathrm{Ma}^{3}$, \\ Lin Kong ${ }^{2,7}$, Jiade J. Lu ${ }^{1,2}$
}

${ }^{1}$ Department of Radiation Oncology, Shanghai Proton and Heavy Ion Center, Shanghai, China; ${ }^{2}$ Shanghai Engineering Research Center of Proton and Heavy Ion Radiation Therapy, Shanghai, China; ${ }^{3}$ Department of Nuclear Medicine, Fudan University Shanghai Cancer Center, Shanghai, China; ${ }^{4}$ Department of Oncology, Shanghai Medical College, Fudan University, Shanghai, China; ${ }^{5}$ Center for Biomedical Imaging, Fudan University, Shanghai, China; ${ }^{6}$ Shanghai Engineering Research Center of Molecular Imaging Probes, Shanghai, China; ${ }^{7}$ Department of Radiation Oncology, Shanghai Proton and Heavy Ion Center, Fudan University Cancer Hospital, Shanghai, China

Contributions: (I) Conception and design: J Hu, L Kong, JJ Lu; (II) Administrative support: L Kong, JJ Lu; (III) Provision of study materials or patients: J Hu, J Gao, W Hu, J Yang, X Qiu, L Kong, JJ Lu; (IV) Collection and assembly of data: J Hu, Z Yang, J Gao, W Hu, J Yang, X Qiu, G Ma; (V) Data analysis and interpretation: J Hu, Z Yang, L Kong, JJ Lu; (VI) Manuscript writing: All authors; (VII) Final approval of manuscript: All authors. Correspondence to: Lin Kong, MD; Jiade J. Lu, MD. Shanghai Proton and Heavy Ion Center, 4365 Kangxin Road, Pudong, Shanghai 201321, China. Email: lin.kong@sphic.org.cn; jiade.lu@sphic.org.cn.

\begin{abstract}
Background: Although carbon-ion radiotherapy (CIRT) may improve outcome for patients with locoregionally recurrent nasopharyngeal carcinoma (LR-NPC), local progression still remains one of the major failure patterns. This suggests an unmet need of markers for predicting disease control after reirradiation and potentially guiding tailored treatment. The purpose of this study was to explore the predictive value of pre-treatment 3'-deoxy-3'-[ $\left.{ }^{18} \mathrm{~F}\right]$ fluorothymidine (FLT)-positron emission tomography (PET) for patients with locally advanced LR-NPC.

Methods: In this retrospective analysis, LR-NPC patients with locally advanced stage (stage III/IV) who received pre-treatment FLT-PET between June, 2015, and August, 2017, were retrospective reviewed and included in this study. OS and local progression-free survival (LPFS) were calculated using the KaplanMeier method. Univariable and multivariable Cox regression analyses of LPFS were performed. FLTderived parameters, including $\mathrm{SUV}_{\max }$, metabolic tumor volume (MTV), and total lesion thymidine (TLT) were examined. The relationship between FLT-derived parameters and mucosal necrosis was tested by the Wilcoxon test.
\end{abstract}

Results: A total of 27 patients with a median follow-up of 31.3 months were included in this analysis. The 2-year OS and LPFS rates were $85.2 \%$ and $47.9 \%$, respectively. In multivariable analysis, except for TLT40\% ( $\mathrm{P}=0.059)$, all pre-treatment MTVs ( $\mathrm{P}=0.040$ for MTV-40\%; $\mathrm{P}=0.021$ for MTV-50\%; $\mathrm{P}=0.026$ for MTV-60\%) and TLTs ( $\mathrm{P}=0.043$ for TLT-50\%; $\mathrm{P}=0.048$ for TLT-60\%) were significantly related to LPFS. Moreover, MTVs and TLTs with various boundaries (except for MTV-40\%) were also associated with the development of mucosal necrosis after CIRT.

Conclusions: In the current study, a significant association between pre-treatment FLT-PET and LPFS was observed in patients with locally advanced LR-NPC. Further investigations are warranted to confirm the predictive role of FLT-PET.

Keywords: 3'-deoxy-3'-[ $\left.{ }^{18} \mathrm{~F}\right]$ fluorothymidine (FLT); locoregionally recurrent nasopharyngeal carcinoma (LRNPC); carbon-ion radiotherapy (CIRT); outcome prediction 
Submitted May 22, 2020. Accepted for publication Aug 21, 2020.

doi: $10.21037 / \mathrm{atm}-20-5214$

View this article at: https://dx.doi.org/10.21037/atm-20-5214

\section{Introduction}

The treatment of locoregionally recurrent nasopharyngeal carcinoma (LR-NPC) is clinically challenging. More than half of LR-NPC patients exhibit locally advanced disease $(1,2)$; however, surgery, brachytherapy, and stereotactic radiosurgery are generally only available to patients with limited tumor volume (3-5). The treatment options for patients with locally advanced disease are therefore limited, with intensity-modulated radiotherapy (IMRT) as the mainstay of treatment.

IMRT as salvage treatment for patients with LR-NPC is clinically difficult. Previous studies showed a 2-year overall survival (OS) rates between $60 \%$ to $70 \%$ after re-irradiation, while the 2-year OS rate for patients with locally advanced disease was around $50 \%(1,2,6)$. In addition, re-irradiation with IMRT has been associated with an increased incidence of severe adverse events (SAE). These SAEs include neurological injury, mucosal necrosis, and subsequent massive hemorrhage, which could significantly compromise patients' quality of life and potentially prove fatal. Previous studies have reported that IMRT-related SAEs accounted for more than $50 \%$ of deaths among LR-NPC patients $(1,6)$.

Carbon-ion radiotherapy (CIRT), which is considered as an emerging advanced radiation technology, has been applied as a potential treatment for recurrent head and neck cancers, including LR-NPC $(7,8)$. Combs et al., showed in a study of 18 patients, that CIRT as re-irradiation achieved a satisfactory disease control with mild acute and late toxicities for recurrent skull base tumors, which is a comparable condition to LR-NPC (7). In our initial study of 75 patients with LR-NPC, the results indicated that CIRT could provide excellent 1-year OS and LPFS rates of $98.1 \%$ and $86.6 \%$ (8). While no patient developed grade $\geq 2$ acute toxicities, and the severe late toxicities were infrequent with only $9.3 \%$ of the patients developed mucosal necrosis. Nevertheless, some patients may still develop local recurrence after CIRT, leading to a dismal outcome. Individualized CIRT dose prescription based on the risk of disease recurrence may aid in achieving a balance between tumor control and treatment-related toxicities. Thus, biomarkers that can predict the outcome of LR-NPC are required.

Three'-deoxy-3'-[ ${ }^{18}$ F] fluorothymidine (FLT) is a newly developed positron emission tomography (PET) tracer used for imaging cellular proliferation in tumors (9). FLT has been suggested as a predictive tool of survival in patients with head and neck cancers (10-16). In a study of 32 patients with head and neck squamous cell carcinoma, Hoshikawa et al. showed that parameters derived from pre-treatment FLT-PET were predictive of locoregional control and OS after surgery and/or radiotherapy (10). In another study of 20 patients, the authors indicated that pretreatment FLT-PET was able to predict the response after induction chemotherapy for patients with newly diagnosed NPC (15); however, the association between FLT-PET and long term survivals were not assessed due to the limited events occurred during the study. The predictive role of FLT for patients who received CIRT was also examined in a study of 13 patients with head and neck mucosal malignant melanoma (13). Although limited by small sample size, the result indicated that FLT-PET could potentially serve as a prognostic tool for CIRT. However, to the best of our knowledge, the predictive potential of FLT-PET has not been investigated in LR-NPC patients treated with CIRT.

This study aimed to examine whether pre-treatment FLT-PET could predict the outcome of patients with locally advanced LR-NPC salvaged by CIRT and be used to guide a tailored treatment plan to improve disease control while reducing treatment-related toxicities. We present the following article in accordance with the STROBE reporting checklist (available at https://atm.amegroups.com/article/ view/10.21037/atm-20-5214/rc).

\section{Methods}

\section{Patients}

In the current retrospective analysis, the medical records of patients with locally advanced (stage III/IV) LR-NPC who received CIRT at Shanghai Proton and Heavy Ion Center (SPHIC) between June, 2015, and August, 2017 were reviewed. Patients were deemed as eligible for study enrolment if they (I) had pathologically confirmed squamous cell carcinoma of the nasopharynx; (II) had undergone FLT-PET/CT scan before CIRT; (III) had failed previous definitive radiotherapy at least 6 months before re-irradiation; (IV) aged 18-80 years; (V) had an Eastern Cooperative 

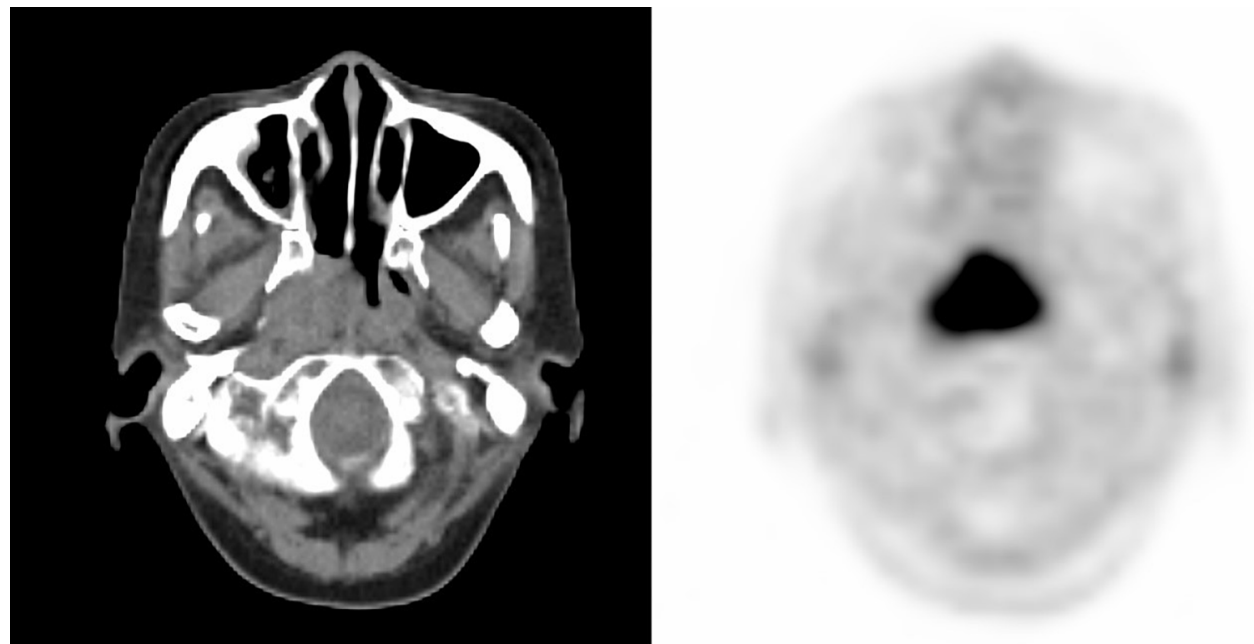

Figure 1 Representative images of FLT-PET/CT for a patient with LR-NPC (rT3N0M0). FLT-PET, 3'-deoxy-3'-[18F]fluorothymidinepositron emission tomography.

Oncology Group (ECOG) performance status of 0-2; (VI) had sufficient major organ functions. Patients were excluded if they (I) had distant metastasis at baseline; (II) were contraindicated to receive MRI for response evaluation; (III) refused to share information for research purpose. Patients who were already participating in one of three preexisting phase I/II clinical trials received CIRT in accordance with the respective study protocols. The remaining patients received CIRT as part of the standard treatment protocol of SPHIC. All patients were re-staged for their recurrent disease according to the 7th edition of the American Joint Committee on Cancer (AJCC) staging classification.

This study was approved by the Institutional Review Board of Shanghai Proton and Heavy Ion Center (No. 200403EXP-01) with a waiver for informed consent. The study was conducted in accordance with the Declaration of Helsinki (as revised in 2013).

\section{FLT-PET/CT scans}

${ }^{18}$ F-FLT was produced with a radiochemical purity above $95 \%$. PET/CT scanning was initiated 1 hour after administration of the tracer (dosage: $7.4 \mathrm{MBq} / \mathrm{kg}$ ) on a Siemens biograph 16 HR scanner (Knoxville, Tennessee, USA). The transaxial intrinsic spatial resolution was $4.1 \mathrm{~mm}$ (full-width at half-maximum) in the center of the field of view. According to the data acquisition procedure, emission images of 3-4 bed positions from head to the base of the lung were recorded in 3-dimensional mode
(2-3 minutes/bed). CT images were acquired for anatomic and attenuation correlation by the setting of $120 \mathrm{kV}, 80-250 \mathrm{~mA}$, pitch 3.6, rotation time 0.5 . The use of contrast agent was not permitted. Representative images of FLT-PET/CT for LR-NPC are illustrated in Figure 1.

\section{FLT-PET analysis}

PET/CT images were independently assessed by two experienced nuclear medicine physicians on a Syngo multimodality computer platform (Siemens, Knoxville, Tennessee, USA). Quantitative analysis of tumor proliferative activity was conducted using standardized uptake value (SUV), with the injected dose, tracer decay and patients' body weight adjusted for. Both the maximum $\left(\mathrm{SUV}_{\max }\right)$ and mean SUV (SUVmean) were recorded by manually selecting a region of interest (ROI) containing the tumor. The metabolic tumor volumes (MTVs) were automatically calculated based on the ROI, with the thresholds set to $40 \%, 50 \%$, and $60 \%$ of the $\mathrm{SUV}_{\max }$. The total lesion thymidine (TLT) was calculated as the product of SUVmean and the corresponding MTV.

\section{Treatment}

CIRT was performed using a technique previously described (8). Briefly, the patient was immobilized in the supine position. CT simulation covering the whole head and neck region was performed at a $1.5-\mathrm{mm}$ cut and fused 
Table 1 The baseline and treatment characteristics of all patients.

\begin{tabular}{lc}
\hline Characteristics & No. of patients (\%) \\
\hline Age & $46[29-70]$ \\
Median [range], year & $21(77.78)$ \\
$<60$ & $6(22.22)$ \\
$\geq 60$ & \\
Gender & $8(29.63)$ \\
Female & $19(70.37)$ \\
Male & \\
Recurrent disease stage & $17(62.96)$ \\
III & $10(37.04)$ \\
IV & \\
Carbon-ion radiotherapy & $57.5[50-63]$ \\
Median dose to GTV [range], GyE & $2.5(2.5-3)$ \\
Median fractionation (range), GyE & $71.88(62.5-81.9)$ \\
Median BED (range), GyE & \\
Chemotherapy & $5(14.81)$ \\
Chemotherapy before CIRT & \\
Chemotherapy concurrent with CIRT & \\
\hline
\end{tabular}

GTV, gross tumor volume; GyE, gray equivalent; BED, biological equivalent dose; CIRT, carbon-ion radiotherapy.

with MRI. The gross tumor volume (GTV) was defined as gross disease detected by physical examination and/or imaging studies, while the clinical target volume (CTV) was defined as GTV plus 5-mm expansion. The CTV was expanded by 3 to $6 \mathrm{~mm}$ to obtain the planning target volume (PTV). This adjustment was performed to address set-up errors and range uncertainties related to CIRT.

Chemotherapy was prescribed at the discretion of the attending radiation oncologist. All patients with locally advanced disease were recommended to undergo induction chemotherapy. Concurrent chemotherapy was not recommended except for participants in the phase I/II trial for evaluating the safety and efficacy of CIRT combined with concurrent chemotherapy (17).

\section{Statistic analysis}

OS was defined as the duration from diagnosis of recurrence to death. Local progression-free survival (LPFS) was defined as the duration from recurrence to local failure or death. The treatment response was evaluated according to the Response Evaluation Criteria in Solid Tumors version 1.1 (18). The OS and LPFS rates were calculated by using the Kaplan-Meier method. Univariable and multivariable analyses of LPFS were conducted using the Cox regression model. In the multivariable analyses, each FLT-derived parameter was treated as continuous variable and analyzed with adjustment for age, gender, and biological equivalent dose (BED) of CIRT to the GTV. In the current study, $22(81.5 \%)$ patients received induction chemotherapy, whereas only $4(14.8 \%)$ patients underwent concurrent chemotherapy. Therefore, multivariable analysis was performed on the patients' data, irrespective of whether they underwent chemotherapy. As the patient population only comprised patients with locally advanced disease (stage III/IV), stage was not included in the multivariable analysis. The relationship between FLT-derived parameters and mucosal necrosis was tested by the Wilcoxon test. $\mathrm{P}$ values $<0.05$ were considered to represent statistical significance. All analyses were performed using R statistical software (version 3.5.1; R Foundation, Vienna, Austria).

\section{Results}

\section{Patients}

A total of 27 patients with locally advanced (recurrent stage III/IV) LR-NPC were included in the analysis. The patients' baseline characteristics and treatment information are detailed in Table 1. Among the 27 patients, 14 patients were recruited to 1 of 3 phase I/II trials at our center and treated according to corresponding prospective protocols, and the rest 13 patients were treated according to the standard treatment protocol of SPHIC.

\section{Survivals and disease control}

The median follow-up period was 31.3 (range, 9-54.6) months. During follow-up, 11 patients died; and 18, 3, and 2 patients developed local, regional and distant failure, respectively. The 2-year OS and LPFS rates were $85.2 \%$ (95\% CI, $72.8-99.7 \%$ ) and $47.9 \%$ (95\% CI, 32.2-71.1\%), respectively (Figure 2).

\section{Predictive values of FLT-derived parameters}

The parameters derived from FLT-PET, including $\mathrm{SUV}_{\text {max }}$, MTVs, and TLTs, are detailed in Table 2. 

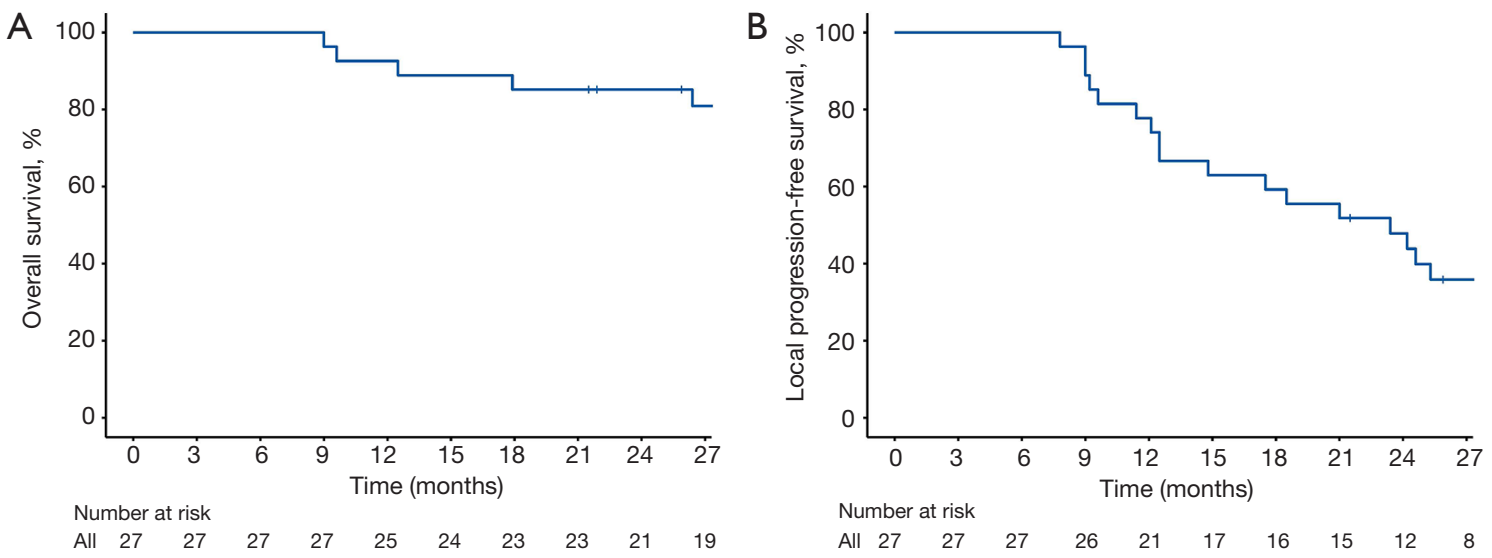

Figure 2 Overall survival (A) and local progression-free survival (B) curves of the cohort. The corresponding 2-year overall survival and local progression-free survival rates were $85.2 \%$ and $47.9 \%$, respectively.

Regression analysis showed that MTVs and TLTs with various boundaries were predictive of LPFS (Table 3). In univariable analysis, increased pre-treatment MTVs ( $\mathrm{P}=0.045$ for $\mathrm{MTV}-40 \% ; \mathrm{P}=0.014$ for $\mathrm{MTV}-50 \% ; \mathrm{P}=0.013$ for MTV-60\%) and TLTs ( $\mathrm{P}=0.048$ for TLT- $40 \%$; $\mathrm{P}=0.027$ for TLT-50\%; $\mathrm{P}=0.024$ for TLT-60\%) were associated with significantly decreased LPFS. Similar results were observed in multivariable analysis. With the exception of TLT- $40 \%$ (HR, 1.02; 95\% CI, 1.00-1.04; $\mathrm{P}=0.059$ ), all pre-treatment MTVs ( $\mathrm{P}=0.040$ for $\mathrm{MTV}-40 \% ; \mathrm{P}=0.021$ for $\mathrm{MTV}-50 \%$; $\mathrm{P}=0.026$ for $\mathrm{MTV}-60 \%)$ and TLTs $(\mathrm{P}=0.043$ for TLT $-50 \%$; $\mathrm{P}=0.048$ for TLT- $60 \%$ ) were significantly related to LPFS. No association was detected between $\mathrm{SUV}_{\text {max }}$ and LPFS.

\section{FLT-derived parameters and mucosal necrosis}

Mucosal necrosis is one of the most commonly observed and challenging toxicities for LR-NPC patients who are salvaged by re-irradiation. Among the 11 patients who died in our study, 1 expired from massive hemorrhage subsequent to mucosal necrosis. We also examined the association between FLT-derived parameters and the development of mucosal necrosis post CIRT. With the exception of MTV-40\%, MTVs and TLTs with various boundaries were significantly associated with necrosis after CIRT (Table 4, Figure 3).

\section{Discussion}

This pilot study is the first to use pre-treatment FLTderived parameters, including MTVs and TLTs, as potential imaging markers for patients with LR-NPC. In multivariable analysis, increased MTVs and TLTs (except for TLT-40\%) were associated with significantly decreased LPFS. Moreover, MTVs (except for MTV-40\%) and TLTs were also predictive of the development of mucosal necrosis after CIRT.

The clinical treatment of LR-NPC patients who have failed definitive radiation therapy is challenging. While various modalities have been applied for the treatment of LR-NPC, IMRT remains as the standard-of-care, especially for locally advanced (stage III/IV disease. However, the outcome after salvage IMRT is poor, with a 2 -year OS rate ranging from $60-70 \%(1,6)$. Re-irradiationrelated toxicities are a major concern in the management of LR-NPC. Re-irradiation with a definitive dose is usually associated with an increased incidence of severe toxicities (such as neurological injuries and mucosal necrosis) that can significantly reduce patients' quality of life or even result in death. Earlier studies on IMRT reported that SAEs were a major cause of death for patients with LRNPC, even at an early stage of the disease. In a study of 77 NPC patients who experienced local failure after IMRT, the authors reported that $50(64.9 \%)$ patients developed SAEs (6). Among the 34 patients who died before the last follow-up, 18 (52.9\%) expired from radiation-induced SAEs. In another study of 60 LR-NPC patients with T1/ $\mathrm{T} 2$ disease, grade 3 or above toxicities were observed in $65 \%$ of the patients (19). CIRT, which is recognized as an advanced radiation technology, was also applied as an attempt to effectively treat patients with LR-NPC. Despite promising OS and acceptable toxicity profiles, 
Table 2 FLT-PET derived parameters

\begin{tabular}{lc}
\hline Parameters & Median (range) \\
\hline SUV $_{\max }$ & $4.29(1.34-8.45)$ \\
MTV-40\% & $5.70(2.26-38.07)$ \\
MTV-50\% & $4.00(1.46-29.97)$ \\
MTV-60\% & $2.58(1.06-23.55)$ \\
TLT-40\% & $14.12(4.80-111.16)$ \\
TLT-50\% & $11.78(1.92-95.3)$ \\
TLT-60\% & $7.53(1.35-79.83)$ \\
\hline
\end{tabular}

FLT-PET, 3'-deoxy-3'-[18F]fluorothymidine-positron emission tomography; SUV, standardized uptake value; MTV, metabolic tumor volume; TLT, total lesion thymidine. some patients are still susceptible to local progression (8). Individualized treatment with tailored dose prescription can achieve a careful balance between disease control and radiation-induced toxicities, thus improving overall outcomes. Therefore, accurate and clinically feasible biomarkers need to be identified.

FLT is a thymidine analogue that was developed for the imaging of tumor cell proliferation (9). One study showed that the retention of FLT was facilitated by its phosphorylation by thymidine kinase 1 (TK1) after its introduction into cells (20). TK1 is a key enzyme in the salvage pathway and is up-regulated during the $\mathrm{S}$ phase of the cell cycle (21). Thus, FLT uptake could be used

Table 3 Univariable and multivariable analysis of LPFS

\begin{tabular}{|c|c|c|c|c|}
\hline Variables & \multicolumn{2}{|c|}{ Univariable analysis } & \multicolumn{2}{|c|}{ Multivariable analysis } \\
\hline $\mathrm{SUV}_{\max }$ & $0.91(0.70-1.18)$ & 0.48 & $0.89(0.67-1.18)$ & 0.420 \\
\hline MTV-40\% & $1.07(1.00-1.14)$ & 0.045 & $1.07(1.00-1.14)$ & 0.040 \\
\hline MTV-50\% & $1.11(1.02-1.20)$ & 0.014 & $1.10(1.01-1.19)$ & 0.021 \\
\hline TLT-40\% & $1.02(1.00-1.04)$ & 0.048 & $1.02(1.00-1.04)$ & 0.059 \\
\hline TLT-50\% & $1.03(1.00-1.06)$ & 0.027 & $1.03(1.00-1.05)$ & 0.043 \\
\hline TLT-60\% & $1.04(1.00-1.07)$ & 0.024 & $1.03(1.00-1.06)$ & 0.048 \\
\hline
\end{tabular}

LPFS, local progression-free survival; HR, hazard ratio; CI, confidence interval; SUV, standardized uptake value; MTV, metabolic tumor volume; TLT, total lesion thymidine.

Table 4 The relationship between FLT-derived parameters and mucosal necrosis

\begin{tabular}{lccc}
\hline & \multicolumn{3}{c}{ Median (range) } \\
\cline { 2 - 3 } Parameters & Patients with necrosis & Patients without necrosis \\
\hline SUV $_{\max }$ & $4.40(3.01-5.15)$ & $4.03(1.34-8.45)$ & 0.61 \\
MTV-40\% & $15.86(4.66-38.07)$ & $5.13(2.26-22.32)$ & 0.085 \\
MTV-50\% & $10.51(3.58-29.97)$ & $3.64(1.46-16.80)$ & 0.047 \\
MTV-60\% & $7.07(2.58-23.55)$ & $2.24(1.06-11.02)$ & 0.040 \\
TLT-40\% & $28.23(14.12-111.16)$ & $10.54(4.80-55.58)$ & 0.047 \\
TLT-50\% & $21.02(11.78-95.30)$ & $7.76(1.92-42.69)$ & 0.047 \\
TLT-60\% & $15.41(9.24-79.83)$ & $5.68(1.35-29.14)$ & 0.047 \\
\hline
\end{tabular}

FLT, 3'-deoxy-3'-[18F]fluorothymidine; SUV, standardized uptake value; MTV, metabolic tumor volume; TLT, total lesion thymidine. 

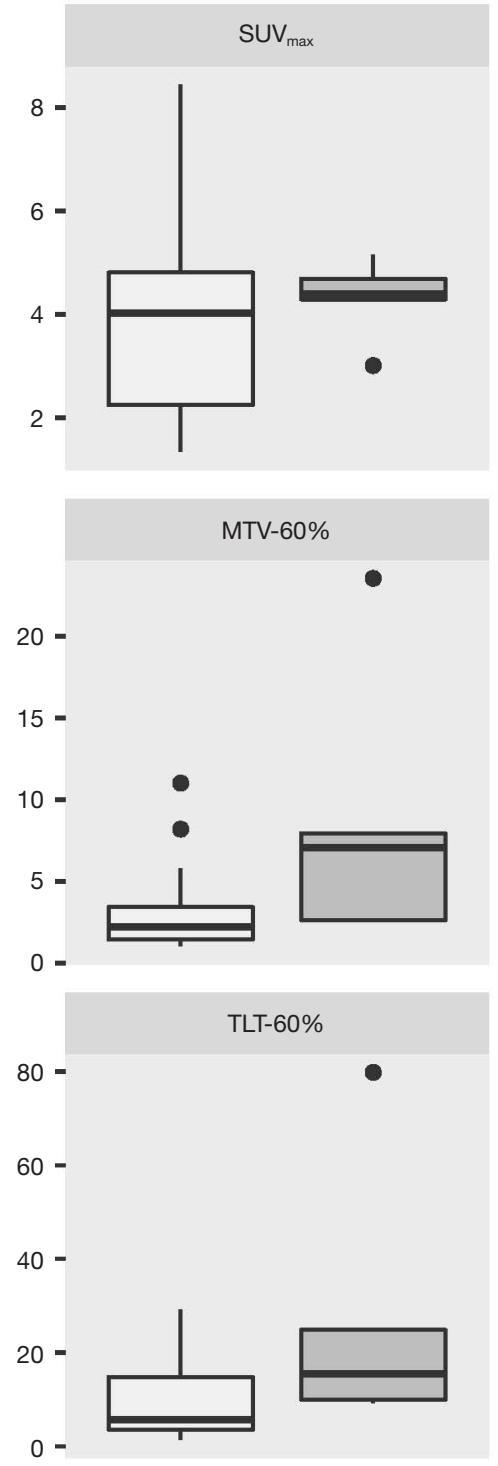
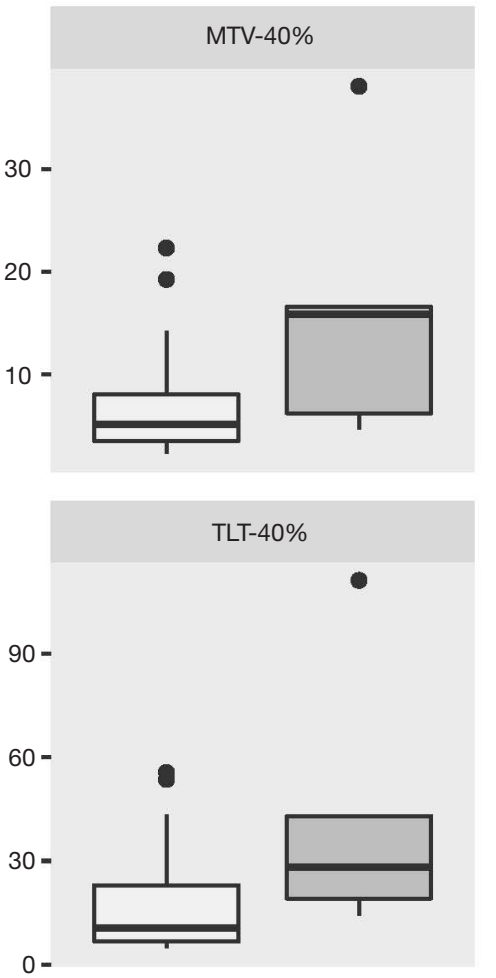

$0-$

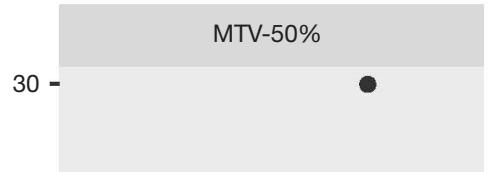

$20-$

$10-$
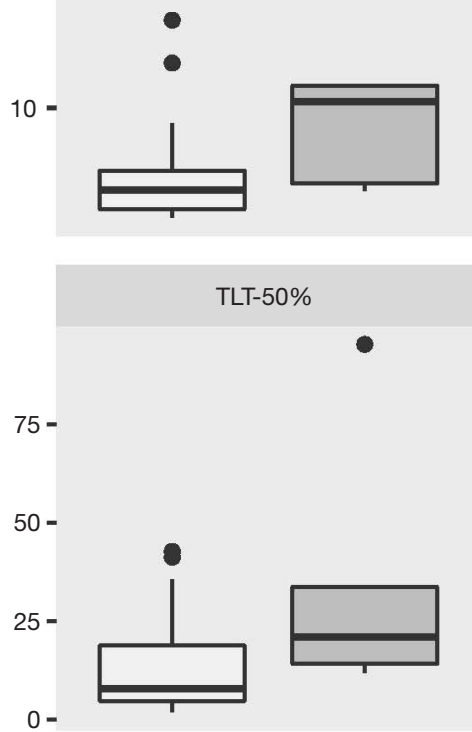

Figure 3 Values of FLT-derived parameters in patients with or without mucosal necrosis. The Wilcoxon test showed that, except for MTV$40 \%(\mathrm{P}=0.085)$, MTVs ( $\mathrm{P}=0.047$ for MTV-50\%; $\mathrm{P}=0.040$ for MTV-60\%) and TLTs ( $\mathrm{P}=0.047$ for TLT-40\%, TLT-50\% and TLT-60\%) with various boundaries were significantly associated with necrosis after CIRT. FLT-PET, 3'-deoxy-3'-[18F]fluorothymidine-positron emission tomography; SUV, standardized uptake value; MTV, metabolic tumor volume; TLT, total lesion thymidine; CIRT, carbon-ion radiotherapy.

to indicate tumor cell proliferation. Although some slow growth tumor may not be detected by FLT-PET because of its typically low uptake, FLT appears to have a higher specificity for imaging tumor proliferation, compared to $\left[{ }^{18} \mathrm{~F}\right]$ fluorodeoxyglucose (FDG) which can be subject to influence from peritumor inflammation $(22,23)$. FLT-PET is particularly useful for patients who has already received antitumor treatment (such as induction chemotherapy). FLT-PET performed before the initiation of anticancer treatment may provide timely guidance for personalized treatment. The predictive value of pre-treatment FLTPET has been examined in head and neck squamous cell carcinoma (HNSCC), a medical condition comparable to nasopharyngeal carcinoma. In a study of 53 HNSCC patients treated with radiotherapy, total lesion proliferation indicated by pre-treatment FLT was significantly related to locoregional control, as were $\mathrm{SUV}_{\max }$ and MTV to OS (16). In this study, 39 patients received induction chemotherapy 
and 14 patients received concurrent chemotherapy. In another prospective study of 48 HNSCC patients, Hoeben et al. demonstrated the association between lower $\mathrm{SUV}_{\text {max }}$ values and significantly improved locoregional control. And a trend of better locoregional control was observed for patients with a smaller visual GTV based on FLT-PET. However, the sample size of this study was not based on power analysis due to the absence of reference data (12). Although another report of 22 newly diagnosed NPC patients demonstrated that pre-treatment FLT-PET was predictive of response after induction chemotherapy, the association between FLT-PET and long-term survival, along with disease control was not assessed, as recurrence or patient death was not observed during the study (15).

Nasopharyngeal mucosal necrosis is one of the most frequently observed SAEs after re-irradiation. It is always associated with refractory headache and a foul odor, and may cause subsequent massive hemorrhage. Previous studies reported it to have an incidence of around $40 \%$ after re-irradiation by photon-based radiotherapy $(1,6)$. Although the frequency of CIRT-related nasopharyngeal mucosal necrosis was substantially reduced, its incidence was still reported in $9.3 \%$ of patients in our initial study (8). Predictions of potential necrosis could be helpful in selecting candidates for re-irradiation and prescribing tailored doses. In the current study, we have identified an association between FLT-PET and necrosis post CIRT. Further studies are necessary to verify this association and to investigate the underlying mechanisms.

A few limitations of this study should be addressed. As this was a pilot study exploring the potential value of FLT-PET in patients with locally advanced LR-NPC, the sample population of 27 limited the number of covariables that could have been included in the multivariable analysis. Nevertheless, we showed a potential association between FLT-derived parameters and LPFS, which could be further verified with larger sample sizes in future studies. Although more feasible for clinical use, cutoff values of FLT-derived parameters were not obtained and examined in the current study due to inter-study variation.

\section{Conclusions}

In this pilot study, parameters derived from pre-treatment FLT-PET showed significant association with the LPFS of locally advanced LR-NPC patients treated with CIRT. Those parameters could serve as potential imaging markers after being validated by further investigations.

\section{Acknowledgments}

Funding: This study was supported by the National Key Research and Development Program of China (project No. 2018YFC0115700), Program of Shanghai Academic/ Technology Research Leader (project No. 18XD1423000), Shanghai Rising-Star Program (project No. 19QB1405500); Science and Technology Development Fund of Shanghai Pudong New Area (project No. PKJ2017-Y51), and Clinical Research Plan of Shanghai Shenkang Hospital Development Center (project No. SHDC12017X13).

\section{Footnote}

Reporting Checklist: The authors have completed the STROBE reporting checklist. Available at https://atm. amegroups.com/article/view/10.21037/atm-20-5214/rc

Data Sharing Statement: Available at https://atm.amegroups. com/article/view/10.21037/atm-20-5214/dss

Conflicts of Interest: All authors have completed the ICMJE uniform disclosure form (available at https://atm. amegroups.com/article/view/10.21037/atm-20-5214/coif). The authors have no conflicts of interest to declare.

Ethical Statement: The authors are accountable for all aspects of the work in ensuring that questions related to the accuracy or integrity of any part of the work are appropriately investigated and resolved. This study was approved by the Institutional Review Board of Shanghai Proton and Heavy Ion Center (No. 200403EXP-01) with a waiver for informed consent. The study was conducted in accordance with the Declaration of Helsinki (as revised in 2013).

Open Access Statement: This is an Open Access article distributed in accordance with the Creative Commons Attribution-NonCommercial-NoDerivs 4.0 International License (CC BY-NC-ND 4.0), which permits the noncommercial replication and distribution of the article with the strict proviso that no changes or edits are made and the original work is properly cited (including links to both the formal publication through the relevant DOI and the license). See: https://creativecommons.org/licenses/by-nc-nd/4.0/.

\section{References}

1. Han F, Zhao C, Huang SM, et al. Long-term outcomes and prognostic factors of re- irradiation for locally recurrent 
nasopharyngeal carcinoma using intensity-modulated radiotherapy. Clin Oncol (R Coll Radiol) 2012;24:569-76.

2. Kong F, Zhou J, Du C, et al. Long-term survival and late complications of intensity-modulated radiotherapy for recurrent nasopharyngeal carcinoma. BMC Cancer 2018;18:1139.

3. Kwong DL, Wei WI, Cheng AC, et al. Long term results of radioactive gold grain implantation for the treatment of persistent and recurrent nasopharyngeal carcinoma. Cancer 2001;91:1105-13.

4. Leung TW, Tung SY, Sze WK, et al. Salvage brachytherapy for patients with locally persistent nasopharyngeal carcinoma. Int J Radiat Oncol Biol Phys 2000;47:405-12.

5. Wei WI. Salvage surgery for recurrent primary nasopharyngeal carcinoma. Crit Rev Oncol Hematol 2000;33:91-8.

6. Kong L, Wang L, Shen C, et al. Salvage IntensityModulated Radiation Therapy (IMRT) for Locally Recurrent Nasopharyngeal Cancer after Definitive IMRT: A Novel Scenario of the Modern Era. Sci Rep 2016;6:32883.

7. Combs SE, Kalbe A, Nikoghosyan A, et al. Carbon ion radiotherapy performed as re-irradiation using active beam delivery in patients with tumors of the brain, skull base and sacral region. Radiother Oncol 2011;98:63-7.

8. $\mathrm{Hu}$ J, Bao C, Gao J, et al. Salvage treatment using carbon ion radiation in patients with locoregionally recurrent nasopharyngeal carcinoma: Initial results. Cancer 2018;124:2427-37.

9. Shields AF, Grierson JR, Dohmen BM, et al. Imaging proliferation in vivo with [F-18]FLT and positron emission tomography. Nat Med 1998;4:1334-6.

10. Hoshikawa H, Yamamoto Y, Mori T, et al. Predictive value of SUV-based parameters derived from pre-treatment (18) F-FLT PET/CT for short-term outcome with head and neck cancers. Ann Nucl Med 2014;28:1020-6.

11. Linecker A, Kermer C, Sulzbacher I, et al. Uptake of (18) F-FLT and (18)F-FDG in primary head and neck cancer correlates with survival. Nuklearmedizin 2008;47:80-5; quiz N12.

12. Hoeben BA, Troost EG, Span PN, et al. 18F-FLT PET during radiotherapy or chemoradiotherapy in head and neck squamous cell carcinoma is an early predictor of outcome. J Nucl Med 2013;54:532-40.

13. Inubushi $M$, Saga T, Koizumi M, et al. Predictive value of 3 '-deoxy-3'-[18F]fluorothymidine positron emission tomography/computed tomography for outcome of carbon ion radiotherapy in patients with head and neck mucosal malignant melanoma. Ann Nucl Med 2013;27:1-10.

14. Arens AI, Troost EG, Hoeben BA, et al. Semiautomatic methods for segmentation of the proliferative tumour volume on sequential FLT PET/CT images in head and neck carcinomas and their relation to clinical outcome. Eur J Nucl Med Mol Imaging 2014;41:915-24.

15. Shi Q, Yang Z, Zhang Y, et al. (18)F-FLT and (18)F-FDG PET/CT in Predicting Response to Chemoradiotherapy in Nasopharyngeal Carcinoma: Preliminary Results. Sci Rep 2017;7:40552.

16. Hoshikawa H, Mori T, Yamamoto Y, et al. Prognostic value comparison between (18)F-FLT PET/CT and (18) F-FDG PET/CT volume-based metabolic parameters in patients with head and neck cancer. Clin Nucl Med 2015;40:464-8.

17. Kong L, Gao J, Hu J, et al. Phase I/II trial evaluating concurrent carbon-ion radiotherapy plus chemotherapy for salvage treatment of locally recurrent nasopharyngeal carcinoma. Chin J Cancer 2016;35:101.

18. Eisenhauer EA, Therasse P, Bogaerts J, et al. New response evaluation criteria in solid tumours: revised RECIST guideline (version 1.1). Eur J Cancer 2009;45:228-47.

19. Tian YM, Guan Y, Xiao WW, et al. Long-term survival and late complications in intensity-modulated radiotherapy of locally recurrent T1 to T2 nasopharyngeal carcinoma. Head Neck 2016;38:225-31.

20. Kong XB, Zhu QY, Vidal PM, et al. Comparisons of anti-human immunodeficiency virus activities, cellular transport, and plasma and intracellular pharmacokinetics of 3'-fluoro-3'-deoxythymidine and 3'-azido-3'deoxythymidine. Antimicrob Agents Chemother 1992;36:808-18.

21. Sherley JL, Kelly TJ. Regulation of human thymidine kinase during the cell cycle. J Biol Chem 1988;263:8350-8.

22. Strauss LG. Fluorine-18 deoxyglucose and false-positive results: a major problem in the diagnostics of oncological patients. Eur J Nucl Med 1996;23:1409-15.

23. Shields AF. PET imaging with 18 F-FLT and thymidine analogs: promise and pitfalls. J Nucl Med 2003;44:1432-4.

(English Language Editors: E. Tan and J. Reynolds)

Cite this article as: $\mathrm{Hu} \mathrm{J}$, Yang Z, Gao J, Hu W, Yang J, Qiu X, Ma G, Kong L, Lu JJ. Pre-treatment FLT-PET parameters as a prognostic tool for patients with locally advanced recurrent nasopharyngeal carcinoma salvaged by carbon-ion radiotherapy: a pilot study. Ann Transl Med 2022;10(22):1196. doi: 10.21037/atm-20-5214 